\title{
Five-colour photometry of OB-stars in the Southern Hemisphere ${ }^{\star}$
}

\author{
C.J. van Houten, Th. Walraven, and J.H. Walraven \\ Sterrewacht Leiden, P.O. Box 9513, 2300 RA Leiden, the Netherlands \\ Received September 10, 1997; accepted December 11, 1998
}

\begin{abstract}
Observations of OB-stars, made in 1959 and 1960 at the Leiden Southern Station near Hartebeespoortdam, South Africa, with the VBLUW photometer attached to the $90 \mathrm{~cm}$ light-collector, are given in this paper. They are compared with photometry obtained by Graham (1968), Walraven \& Walraven (1977), Lub \& Pel (1977) and Van Genderen et al. (1984). Formulae for the transformation of the present observations to those of Walraven \& Walraven (1977) and Lub \& Pel (1977) are given.
\end{abstract}

Key words: stars, early type - stars, fundamental parameters (colours)

\section{Introduction}

The $90 \mathrm{~cm}$ light-collector of the Leiden Observatory, situated at the Leiden Southern Station near Hartebeespoortdam, South Africa, from 1958 till 1978, has been used almost exclusively in combination with the simultaneous five-colour photometer, designed by the second author of this paper. A description of this photometer can be found in Walraven \& Walraven (1960), and a discussion of the photometry based on observations with this photometer is given by Lub \& Pel (1977). Many programmes carried out with this instrument have been published, but the first observational programme made with this photometer, the OB-star programme observed by the second author in 1959 and 1960, is still unpublished. The 1959 observations were reduced completely, shortly after they were made, by the second author.The 1960 observations, as far as they are available in Leiden, were reduced by the first author, who also wrote the text of this publication.

Send offprint requests to: C.J. van Houten, e-mail: vhouten@strw.leidenuniv.nl

* Table 4 is only available in electronic form at the CDS via anonymous ftp to cdsarc.u-strasbg.fr (130.79.128.5) or via http://cdsweb.u-strasbg.fr/Abstract.html

\section{Reduction}

The reduction of the 1959 material was made by means of the electronic computer of the central computing institute of the Leiden University. In this part of the reduction the authors were assisted by Mr. L. Maitimo. The 1960 observations were reduced by hand. Comparison with the 1959 material showed, for most of the 1960 material, systematic differences in the $V$ colour, depending on the $V-B$ colour index. This was caused by the use of a photomultiplier for the $V$ channel with a colour sensitivity different from the one used in 1959. From comparisons between stars observed both in 1959 and in 1960 the following relation between $V(1959)$ and $V(1960)$ could be derived:

$V(1959)=V(1960)-0.254\{V(1960)-B(1960)\}$.

The Walraven VBLUW colour system is constructed such that it is independent of the photomultipliers used, except for the $V$ channel, where the red edge is determined by the photomultiplier used. Expression (1) was used to transfer the 1960 material to the 1959 photometric system. After applying this correction, it turned out that still systematic differences between 1959 and 1960 existed. In order to combine the 1959 and the 1960 results the latter were reduced to the 1959 system by applying corrections to the observations made in 1960. It turned out that a still better agreement with the 1959 observations could be obtained if these systematic differences were determined from and applied to the individual nights. In this way the values listed in Table 4 were obtained. To give an impression of the systematic corrections applied to the 1960 observations, the correction applied to the $B-U$ colour index is -0.007 and to the $U-W$ colour index amounts to +0.012 . The other corrections were all considerably smaller; they amount to 0.002 on the average. These corrections to $B-U$ and $U-W$ are found again in a comparison of OB star observations made in 1965 and 1968 by the first author (van Houten, unpublished) with the 1959 values given here. Its origin is unknown.

It was assumed that the photomultiplier used in the $V$ channel in 1959 was the same as the one used since 1965. 
However, comparisons of the values given in this paper with a compilation by Dr. E. Brinks (unpublished) of observations in the Lub \& Pel (1977) system clearly showed a colour term in the $V-B$ colour indices. The relation found is

$(V-B)_{\mathrm{st}}=(V-B)_{59}-0.076(V-B)_{59}-0.003$.

Here $(V-B)_{\text {st }}$ refers to the Lub \& Pel colour system and $(V-B)_{59}$ to the system used in this publication. A corresponding formula is valid for the $V$ channel. It can be expected that a similar transformation can be found from a comparison of the present material with Walraven \& Walraven (1977). The relation actually found is slightly different:

$(V-B)_{77}=(V-B)_{59}-0.055(V-B)_{59}+0.001$.

Here $(V-B)_{77}$ refers to the observations published in Walraven \& Walraven (1977). The cause of the difference between (2) and (3) is probably that Walraven \& Walraven (1977) was not corrected for the transformation found by Lub \& Pel (1977):

$(V-B)_{\mathrm{st}}=(V-B)_{77}-0.026(V-B)_{77}-0.002$.

If this is true, (4) can be derived from (2) and (3). Adding (3) and (4) yields:

$(V-B)_{\mathrm{st}}=(V-B)_{59}-0.079(V-B)_{59}$

which is, within the limits of accuracy, equal to (2). It follows that for a transformation of the observations presented here to the standard system of Lub \& Pel (1977) Eq. (2) should be used. Transformation of Walraven \& Walraven (1977) to the same standard system needs Eq. (4).

In Table 4 the original 1959 reduction is listed in four decimals. This is more than the observational accuracy warrants, but the reduction was made in this way and it was judged an unnecessary loss of time to reduce them to a three-decimal value. All additions and corrections to this list were made with three-decimal values. Thus threedecimal values in Table 4 mean that an error in the original table has been corrected, that the observations in 1959 were combined with observations in 1960 or that the star was observed in 1960 only. These remarks are meant to explain why some entries in Table 4 have four decimals and other have only three. They are not meant to indicate which observations were made in 1959 and which in 1960. Readers who want to obtain this information can request them from the first author.

\section{The standard stars}

The values for the standard stars, derived in this investigation, are collected in Table 1, together with their (internal) mean errors. The average value of the standard deviation of one observation is 0.008 for $V$ and 0.004 for the colour indices. All quantities here are expressed in logarithms of intensity, in conformity with the usual practice in the Walraven VBLUW colour system. In Table 1 the symbol $\mathrm{n}$ denotes the number of observations used; the other column headings are self-explanatory. Comparisons with standard star values given in other publications show reasonable agreement, with the exception that the derived intensity of HD 210934 in the U channel is approximately 0.015 too bright in 1959 .

\section{Comparisons with other VBLUW observations}

To judge the accuracy of the photometry given here it is important to compare the present values with lists of observations published earlier, and which have stars in common with our Table 4. Four of such lists were compared: 1. Walraven \& Walraven (1977) (denoted here as WW77) published a list of VBLUW measurements on southern stars. They are not all OB-stars. This list has 80 stars in common with the present material. The average differences, defined as: Table 4 minus WW77, are given in Table 3 . The $V-B$ values of 1959 were corrected by means of Eq. (3). The material in W77 was observed in (approximately) 1961-1962 and is thus closest in time to the material presented here.

2. Graham (1968) published the results of an extensive investigation of OB-stars in Carina, observed in 1964. Average differences: Table 4 minus Graham (1968) are given in Table 3 .

3. Pel (1976) and Lub \& Pel (1977) have 23 stars in common with our material. Their material was collected in 1971 and 1972. The average differences are again shown in Table 3 ; the sign convention is as for the comparisons 1 and 2 . For this comparison our $V$ and $V-B$ values were corrected with expression (2).

4. Van Genderen et al. (1984), observing the Sco OB1 association, have 51 stars in common with the present material. Comparison with the 1959 values at first showed rather large differences. Van Genderen et al. used the star HD 151515 as a local substandard for their photometry. This star was used as a standard star by the first author in his (unpublished) survey of southern OB-stars, extending from 1965 to 1978 . The average of the calibrations of HD 151515 made by the first author in 1969, 1974 and 1977 is entered in Table 2, together with the values used by Van Genderen et al. (1984). Using the 1969-1977 calibration instead of the one used by Van Genderen et al. considerably reduced the difference with the 1959 material. It is the difference with the latter values which is listed in Table 3 . Inspection of Table 3 shows that the comparison of our material with Walraven \& Walraven (1977), Pel (1976) and Lub \& Pel (1977) and Van Genderen et al. (1984), after applying the correction of these data, mentioned above, all show about the same pattern, whereas 
Table 1. Adopted values for the primary standards, with their mean errors

\begin{tabular}{llrrrrr}
\hline HD & $n$ & $V$ & $V-B$ & $B-U$ & $U-W$ & $B-L$ \\
\hline 104337 & 220 & +0.6430 & -0.0645 & -0.0084 & -0.0147 & -0.0197 \\
& & \pm 8 & \pm 3 & \pm 3 & \pm 5 & \pm 3 \\
144470 & 400 & +1.1735 & +0.0014 & -0.0010 & +0.0002 & -0.0003 \\
& & \pm 3 & \pm 2 & \pm 2 & \pm 3 & \pm 2 \\
164402 & 328 & +0.4553 & +0.0078 & -0.0346 & +0.0008 & -0.0151 \\
& & \pm 3 & \pm 2 & \pm 2 & \pm 3 & \pm 2 \\
178175 & \multirow{2}{*}{162} & +0.5322 & -0.0134 & +0.0701 & +0.0212 & +0.0169 \\
& & \pm 6 & \pm 3 & \pm 3 & \pm 3 & \pm 3 \\
210934 & \multirow{2}{*}{109} & +0.5786 & -0.0386 & +0.2154 & +0.0528 & +0.0662 \\
& & \pm 7 & \pm 4 & \pm 5 & \pm 8 & \pm 5 \\
\hline
\end{tabular}

Table 2. Calibration of HD 151515 by Van Genderen et al. (1984) and Van Houten (unpublished) and the corrections applied to the photometry of Van Genderen et al. (1984)

\begin{tabular}{lrrrrr}
\hline & $V$ & $V-B$ & $B-U$ & $U-W$ & $B-L$ \\
\hline Van Houten & -0.119 & 0.079 & -0.001 & 0.032 & 0.014 \\
Van Genderen et al. & -0.126 & 0.069 & 0.003 & 0.029 & 0.018 \\
correction terms & +0.007 & +0.010 & -0.004 & +0.003 & -0.004 \\
\hline
\end{tabular}

Table 3. Comparison of the average differences between the present material and four other surveys; in the sense this survey minus others. The internal accuracy of each entry is about 0.001

\begin{tabular}{lrrrrr}
\hline & $\Delta V$ & $\Delta(V-B)$ & $\Delta(B-U)$ & $\Delta(U-W)$ & $\Delta(B-L)$ \\
Walraven \& Walraven (1977) & -0.003 & +0.001 & -0.009 & +0.005 & -0.005 \\
Pel (1976), Lub \& Pel (1977) & -0.005 & +0.000 & -0.012 & +0.010 & +0.001 \\
Van Genderen et al. (1984) & 0.000 & 0.000 & -0.009 & +0.007 & -0.001 \\
average & -0.002 & +0.001 & -0.009 & +0.007 & -0.002 \\
Graham (1968) & +0.002 & +0.001 & +0.003 & +0.022 & +0.007 \\
\hline
\end{tabular}

the comparison with Graham (1968) is different, especially in the ultraviolet. The differences can be interpreted such that in the 1959 material the $U$ channel intensity was systematically 0.008 too bright. This is the same effect as found for the standard star HD 210934, although in the latter case the excess is larger. This suggests that the adoption of an incorrect value of the $U$ channel intensity of one of the standard stars introduced a systematic effect in the colours of the program stars. If this is correct the systematic error can be expected to be dependent on the right ascension of the stars, since for stars with a right ascension very different from that of HD 210934, the latter can have played no part in their photometry. The comparison with WW77, which extends over all right ascensions, shows this indeed to be the case. For stars which have $2^{\mathrm{h}}<\alpha<10^{\mathrm{h}}$ no systematic effect is present, whereas the other stars have their 1959 brightness in the $U$ channel about 0.010 too bright. Using the spectral classification schemes published by Walraven \& Walraven (1960) it is seen that this effect will introduce errors in classification of one tenth of a spectral class, and of about half a magnitude in $M_{v}$. Obviously it would have been better to reduce the 1959 material anew, with improved values of the standard stars, but the material is so large that the amount of work involved is prohibitive, and if the systematic effect derived above is taken into account the data published here are thought to be still useful.

The values in Table 4 were not corrected by means of Eq. (2) or otherwise. In all comparisons of Table 3 the values of the primary standard stars from Table 1 are not included. The standard deviation of one difference in Table 3 is, on the average, $\Delta \log I=0.011$. If it is supposed that the accuracy of all series is equal, then this accuracy amounts to $\Delta \log I=0.008$, or 0.02 magnitudes.

\section{The main table}

Table 4 contains the result of the 1959 and part of the 1960 campaigns. The stars are denoted by their HD number, or BD number if north of $\delta=-23^{\circ}$, or by their Co D number if south of this limit. For the close visual binaries HD 36861/36862, HD 120709/120710 and HD 147934/147935 the intensities and magnitudes given are the combined values for the pair.

Following a suggestion by the referee, and with a view towards making Table 4 more useful, Dr. J. Lub at Leiden Observatory added in the third column a simple code indicating whether the data were taken in 1959, 1960 or both years: 10, 01 or 11 respectively. He also provided a further 
two columns in which the $V$ and $(B-V)$ colours on the Johnson system are given, based upon the transformations published by WW77.

Acknowledgements. The authors acknowledge with thanks Mr. L. Maitimo's share in the reduction of the observations. Many thanks are due to Dr. A.M. van Genderen for several useful remarks on an earlier version of this paper. Many thanks are due to an unknown refeee. His remarks led to a considerable improvement of this paper.

During the preparation of this paper Mrs. J.H. Walraven died. It seems here a fitting place to emphasize how essential her contribution was to the many observational and instrumental projects of her husband, the second author of this paper. She took an active part in the construction of optical instruments by grinding and polishing mirrors and lenses, she assisted in the observations and took a major share in their reductions. Without her such a large production of observations would not have been possible.

\section{References}

van Genderen A.M., Bijleveld W., van Groningen E., 1984, A\&AS 58, 537

Graham J.A., 1968, Bull Astr. Inst. Neth. Suppl. 2, 397

Lub J., Pel J.W., 1977, A\&A 54, 137

Pel J.W., 1976, A\&AS 24, 413

Walraven T., Walraven J.H., 1960, Bull. Astr. Inst. Neth. 15, 67

Walraven T., Walraven J.H., 1977, A\&AS 30, 245 